\title{
HIV protease inhibitors: a review of molecular selectivity and toxicity
}

This article was published in the following Dove Press journal:

HIVIAIDS - Research and Palliative Care

8 April 2015

Number of times this article has been viewed

\section{Zhengtong Lv* \\ Yuan Chu* \\ Yong Wang}

Department of Immunology, School of Basic Medical Science, Xiangya School of Medicine, Central South University, Changsha, Hunan, People's Republic of China

*Both authors contributed equally to this work
Correspondence: Yong Wang Department of Immunology, School of Basic Medical Science, Xiangya School of Medicine, Central South University, Tongzipo Road 172, Changsha, Hunan 4I00I3, People's Republic of China

Email wangyong@csu.edu.cn
Abstract: Highly active antiretroviral therapy (HAART) is recognized as the most effective treatment method for AIDS, and protease inhibitors play a very important role in HAART. However, poor bioavailability and unbearable toxicity are their common disadvantages. Thus, the development of safer and potentially promising protease inhibitors is eagerly needed. In this review, we introduced the chemical characteristics and associated side effects of HIV protease inhibitors, as well as the possible off-target mechanisms causing the side effects. From the chemical structures of HIV protease inhibitors and their possible off-target molecules, we could obtain hints for optimizing the molecular selectivity of the inhibitors, to provide help in the design of new compounds with enhanced bioavailability and reduced side effects.

Keywords: off-target, side effect, glucose transporter-4, proteasome

\section{Introduction}

Since the discovery of HIV, 26 anti-HIV compounds have been approved by the US Food and Drug Administration (FDA). Among these compounds, ten are HIV protease inhibitors. The combination therapy of HIV protease inhibitors, reverse transcriptase inhibitors, and/or an integrase inhibitor, referred to as highly active antiretroviral therapy (HAART), is the current most effective AIDS therapy. The AIDS-related mortality has dropped sharply, and AIDS has gradually become a controllable, chronic disease. Based on global AIDS response progress reporting, there are nearly 13 million people receiving antiretroviral therapy, and this number could reach 16 million by $2015 .{ }^{1}$ HIV protease inhibitor is one of the most important components in the combination therapy. In the preferred antiretroviral combination regimens, protease inhibitorbased therapy has returned a lower level of resistance compared with non-nucleoside reverse-transcriptase inhibitor (NNRTI)-based therapy. ${ }^{2}$ However, the need for lifelong treatment and the frequently associated side effects of HIV protease inhibitors severely hurt patient compliance, which is one of the obstacles in the treatment of HIV/AIDS patients. Although the toxic effects of HIV protease inhibitors could result from drugdrug interactions and overdose, the off-target adverse drug effects of therapeutic doses is a major concern in drug design.

In the HIV life cycle, protease is an essential element for viral maturation. The HIV protease is a homodimeric aspartyl protease, and each monomer is composed of 99 amino acid residues with a catalytic Asp at position 25 (Figure 1). HIV-1 protease cleaves Gag and Gag-Pol polyprotein precursor encoded by the HIV-1 virus genome at nine processing sites to produce mature active proteins. The Pol polyproteins is first 


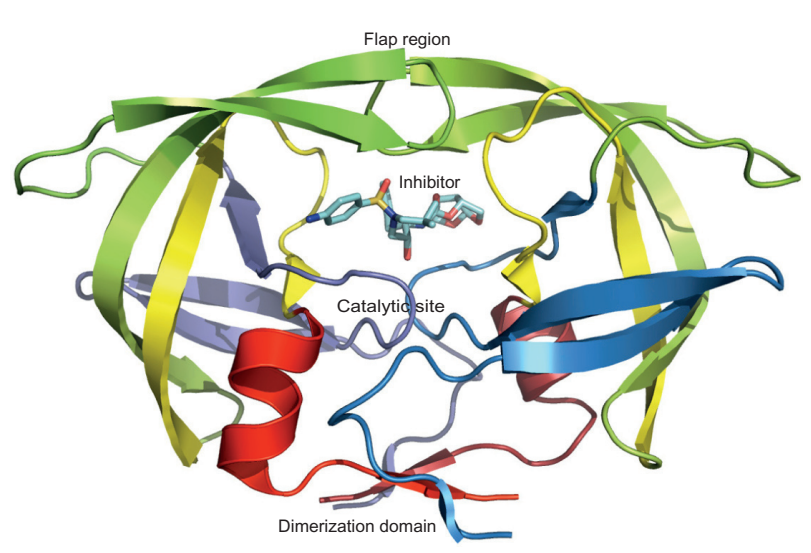

Figure I The HIV-I protease structure in complex with an inhibitor.

cleaved off from the Gag-Pol polyproteins and then further digested into protease, reverse transcriptase (p51), RNase $\mathrm{H}$ (p15), and integrase. The active site is not fully exposed, being covered by two flexible $\beta$-hairpin flaps. The flaps need to open to allow the substrates to access the active site. The HIV-1 protease enzyme activity can be inhibited by blocking the active site of the protease.
The indispensable role of HIV protease in viral maturation makes it a popular target for drug design. A large number of solved HIV protease protein structures have greatly facilitated the design of new and improved inhibitors. There are ten HIV protease inhibitors approved by the FDA; those inhibitors include: saquinavir, indinavir, ritonavir, nelfinavir, amprenavir, fosamprenavir, lopinavir, atazanavir, tipranavir, and darunavir (Figure 2). Unfortunately, most of the inhibitors are accompanied by side effects in long-term treatment. The most common side effects are HIV protease inhibitor-induced metabolic syndromes, such as dyslipidemia, insulin-resistance, and lipodystrophy/lipoatrophy, as well as cardiovascular and cerebrovascular diseases. ${ }^{3-6}$ Protease inhibitor monotherapy is associated with a mild improvement in body fat distribution. ${ }^{78}$ However, regarding the serious adverse events of antiretroviral treatments, no significant between-group differences were found between HIV protease inhibitor monotherapy and the combination of protease inhibitors with the HIV integrase inhibitor raltegravir or nucleoside reverse transcriptase inhibitors (NRTIs), ${ }^{9}$

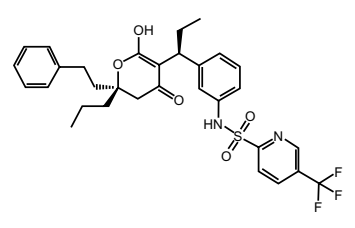

Tipranavir

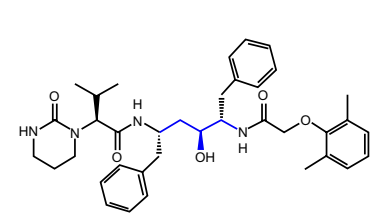

Lopinavir<smiles>CC(C)CN(CC(O)C(Cc1ccccc1)NC(=O)OCC1CO[C@]2(C)OCC[C@@]12F)S(=O)(=O)c1ccc(N)cc1</smiles>

Darunavir

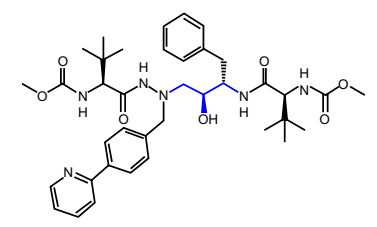

Atazanavir

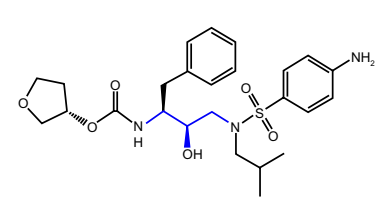

Amprenavir

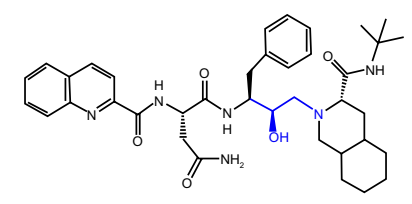

Saquinavir

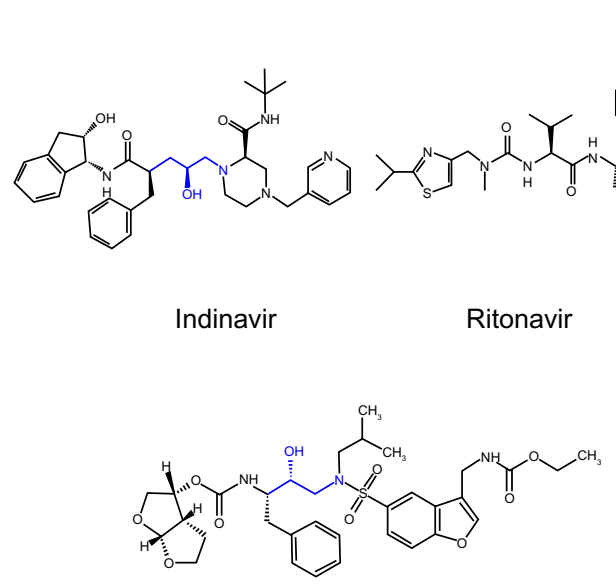

SPI-256

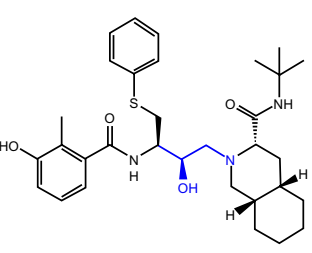

Nelfinavir

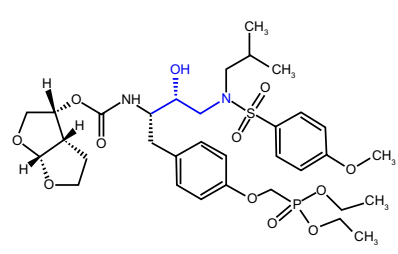

GS 8374

Figure 2 Chemical structures of the HIV protease inhibitors. 
indicating that HIV protease inhibitors may be responsible for the most serious adverse effects.

The FDA-approved HIV protease inhibitors share same structural similarities and a similar binding pattern, which may cause some of the common side effects of the protease inhibitor-containing regimens.

\section{Saquinavir}

Saquinavir (brand name: Invirase), developed by F. HoffmannLa Roche Ltd (Basel, Switzerland), was the first FDAapproved HIV protease inhibitor used in the treatment of patients with AIDS (in 1995). The original design for the precursor of saquinavir comprised a proline at the $\mathrm{P}^{\prime}$ site and a phenylalanine at the $\mathrm{P} 1$ site. The rationale is that HIV-1 protease cleaves the substrate between a phenylalanine and a proline, while mammalian proteases do not cleave substrates containing proline at the $\mathrm{P}^{\prime}$ ' site. In the final structure of saquinavir, the proline was replaced by a $\mathrm{z}(\mathrm{S}, \mathrm{S}, \mathrm{S})$-decahydroisoquinoline- 3-carbonyl (DIQ) group to enhance the inhibitory potency. The carbonyl of the DIQ group contacts the bridging water molecule, which interacts with the inhibitor and the flaps of HIV-1 protease. ${ }^{10}$ The mean $50 \%$ effective concentration $\left(\mathrm{EC}_{50}\right)$ of saquinavir against HIV-1 in MT4 cells is $37.7 \mathrm{nM} .{ }^{11}$ The adult dose is twice daily saquinavir $1,000 \mathrm{mg}$ in combination with ritonavir $100 \mathrm{mg}$. Few side effects related to saquinavir have been reported. ${ }^{12}$ However, saquinavir is not a preferred protease inhibitor regimen due to its low bioavailability. ${ }^{13}$ The most common clinically significant saquinavir resistance mutations are 48VM, 54VTALM, 82AT, 84V, 88S, and 90M.

\section{Indinavir}

Indinavir (brand name: Crixivan) was developed by Merck \& Co, Inc., (Whitehouse Station, NJ, USA) and approved in 1996. The $\mathrm{EC}_{50}$ of indinavir is $\sim 5.5 \mathrm{nM}$. An advantage of indinavir is its effective inhibition of both HIV-1 and HIV-2, while the disadvantage is the quick decrease in the concentration of circulating indinavir. The low plasma concentration of indinavir usually leads to treatment failures. ${ }^{14}$ In addition, the low solubility of indinavir may result in the development of kidney stones. Furthermore, indinavir could act as a competitive inhibitor of the cytoplasmic glucose binding site of GLUT4, ${ }^{15}$ and lipodystrophy syndrome is strongly associated with indinavir. ${ }^{16}$ Further, indinavir has a short acting time and requires a dosage of $800 \mathrm{mg}$ every 8 hours. For these reasons, indinavir has been replaced by second-generation protease inhibitors. The most common clinically significant indinavir resistance mutations are 32I, 46IL, 47V, 54VTALM, 76V, 82ATFS, 84V, 88S, and 90M.

\section{Ritonavir}

Ritonavir (brand name: Norvir), developed by Abbott Laboratories (Abbott Park, IL, USA) and approved by the FDA in 1996, was originally designed as an HIV protease inhibitor $\left(\mathrm{EC}_{50} \sim 25 \mathrm{nM}\right)$, but it was found later that ritonavir boosts the circulating concentration of other HIV protease inhibitors by inhibiting cytochrome P450 3A4. ${ }^{17}$ Regarding the molecule structure, the isopropyl thiazolyl P3 group in ritonavir is longer than that in other FDA-approved HIV protease inhibitors. As a remarkably potent inhibitor of P450 3A4, a subtherapeutic dose of ritonavir has been used to boost the plasma concentration of the second generation of HIV protease inhibitors, since HIV protease inhibitors are extensively metabolized by cytochrome P450 3A4. ${ }^{18}$ Ritonavir inhibits cytochrome P450 3A4 isoenzyme and prevents the metabolism of other protease inhibitors. Normally, boosted HIV protease inhibitors improve the side-effect and toxicity profile of HAART regimens. However, cytochrome P450 3A4 polymorphism, which encodes a nonfunctional protein, affects the metabolism of boosted HIV protease inhibitors, causing a higher plasma concentration of HIV protease inhibitors and increased toxicity. ${ }^{19}$ Ritonavir-boosted protease inhibitor regimens require less frequent dosing, which benefits patients. However, higher doses could lead to hyperlipidemia in AIDS patients or healthy volunteers taking ritonavir. ${ }^{20}$

\section{Nelfinavir}

Nelfinavir (brand name: Viracept) was developed by Agouron Pharmaceuticals (Pfizer, Inc., New York, NY, USA) and approved in 1997. One terminus of the nelfinavir molecule has the same DIQ group as saquinavir. The other terminus of nelfinavir contains a 2-methyl-3-hydroxybenzamide group. The S-phenyl group at the P1 site was designed to magnify the potency of this inhibitor. The $\mathrm{EC}_{50}$ of nelfinavir is $30-60 \mathrm{nM}$. The preferred regimen of nelfinavir is $1,250 \mathrm{mg}$ orally, twice a day. The most common side effects related to nelfinavir are diarrhea and nausea. ${ }^{21}$ The clinically significant nelfinavir resistance mutations are $30 \mathrm{~N}, 33 \mathrm{~F}, 46 \mathrm{IL}, 47 \mathrm{~V}, 48 \mathrm{VM}$, 54VTALM, 82ATFS, 84V, 88DS, and 90M.

\section{Lopinavir}

Lopinavir (brand name: Kaletra), containing lopinavir and ritonavir, from Abbott Laboratories, was approved by the FDA in 2000 and was developed as a ritonavir-based agent. 
The core region of lopinavir, a hydroxyethylene dipeptide isostere, is the same as that of ritonavir. The P2 and P2' group are altered in lopinavir relative to ritonavir. The 5-thiazolyl P2 group of ritonavir is replaced by a phenoxyacetyl group, and the 2-isopropylthiazolyl $\mathrm{P} 2$ ' group of ritonavir is replaced by a six-member cyclic urea. In general, the new P2 and P2' groups are smaller in order to decrease the contact with highly variable residues at the 82 site of HIV-1 protease. ${ }^{22}$ The substitution of the $\mathrm{P} 2$ and $\mathrm{P} 2^{\prime}$ groups improves the inhibitory potency of lopinavir against the drug-resistant variants of HIV-1 protease. ${ }^{22}$ Lopinavir inhibits HIV protease activity, with the $\mathrm{EC}_{50}$ of $\sim 17 \mathrm{nM}$. The dosage for adult patients is lopinavir $400 \mathrm{mg}$ plus ritonavir $100 \mathrm{mg}$ orally, twice a day. Lopinavir induces insulin resistance and could cause systemic hypersensitivity syndromes and Achilles tendinopathy. ${ }^{23-25}$ The most common clinically significant lopinavir resistance mutations are 32I, 33F, 46IL, 47VA, 48VM, 50V, 54VTALM, $76 \mathrm{~V}, 82 \mathrm{ATFS}, 84 \mathrm{~V}$, and $90 \mathrm{M}$.

\section{Amprenavir}

Amprenavir (brand name: Agenerase), developed by Vertex Pharmaceuticals Incorporated, (Boston, MA, USA) and approved in 1999, has a benzyl group at the P1 site and an isobutyl group at the $\mathrm{P} 1^{\prime}$ site. The $\mathrm{P} 1^{\prime}$ group and the phenyl amide $\mathrm{P} 2$ ' group are connected by a sulfonamide. The asymmetry of the $\mathrm{P} 1$ and $\mathrm{P}^{\prime}$ groups may favor the internal pseudosymmetry of HIV-1 protease. Amprenavir contains fewer chiral centers than do previous HIV protease inhibitors. This improvement simplifies the chemical synthesis and increases the oral availability. ${ }^{26}$ The dosage of amprenavir is $1,200 \mathrm{mg}$ orally, twice a day. Amprenavir is less effective on HIV-2 protease than on HIV-1 protease. ${ }^{27}$ The $\mathrm{EC}_{50}$ of amprenavir has ranged from 12-80 nM. Amprenavir and its prodrug, fosamprenavir, cause the side effect of benign skin rash.

\section{Fosamprenavir}

Fosamprenavir (brand name: Lexiva), developed by GlaxoSmithKline plc, (Brentford, UK) and Vertex Pharmaceuticals Incorporated, was approved by the FDA in 2003. Fosamprenavir is the phosphate ester prodrug of amprenavir. The body metabolizes fosamprenavir to form the active ingredient, amprenavir. That metabolization increases the duration that amprenavir is available, making fosamprenavir a slowrelease version of amprenavir and, thus, reducing the number of pills required versus standard amprenavir. ${ }^{28}$ The recommended dose is fosamprenavir $1,400 \mathrm{mg}$ in combination with ritonavir $100 \mathrm{mg}$ orally, twice a day. Clinical studies demonstrated that fosamprenavir has a safer profile compared with amprenavir. ${ }^{29,30}$ The most common clinically significant fosamprenavir resistance mutations are 32I, 33F, 46IL, 47VA, 50V, 54VTALM, 76V, 82ATFS, 84V, and 90M.

\section{Atazanavir}

Atazanavir (brand name: Reyataz) developed by BristolMyers Squibb Co. (Princeton, NJ, USA) and approved in 2003, is an aza-dipeptide analog, which exhibits potent anti-HIV activity. The $\mathrm{EC}_{50}$ of atazanavir in cell culture is 2.6-5.3 nM. A unique characteristic of atazanavir is the presence of a large phenylpyridyl P1 group that is asymmetric relative to its benzyl $\mathrm{P}^{\prime}$ group. Atazanavir shows good oral bioavailability. ${ }^{31}$ Thus, the benefit of this is once-a-day dosing with atazanavir $300 \mathrm{mg}$ plus ritonavir $100 \mathrm{mg}$. Fewer side effects are associated with atazanavir than with other protease inhibitors. ${ }^{32,33}$ Atazanavir has no effect on insulin sensitivity and serum lipid concentration. ${ }^{23,34}$ However, a significantly higher incidence of proximal tubulopathy was observed among atazanavir-treated patients. ${ }^{35,36}$ The most common clinically significant atazanavir resistance mutations are 32I, 33F, 46IL, 47V, 48VM, 50L, 54VTALM, 82ATFS, $84 \mathrm{~V}, 88 \mathrm{~S}$, and 90M.

\section{Tipranavir}

Tipranavir (brand name: Aptivus) developed by Boehringer Ingelheim GmbH (Ingelheim, Germany), is the only nonpeptidomimetic HIV protease inhibitor and received approval from the FDA in 2005. Due to dissimilarities with other HIV protease inhibitors, tipranavir encounters a relatively different HIV-1 protease resistance profile. ${ }^{37,38}$ An innovation in the tipranavir design is the functional substitution of the bridging water molecule connecting the inhibitor and protease flaps. The lactone oxygen atom of the dihydropyrone ring of tipranavir interacts directly with the Ile50 residues in the flap region of the HIV-1 protease. The direct interaction stabilizes the protease-inhibitor complex. Tipranavir inhibits the HIV-1 protease that has developed resistance to other protease inhibitors. Though the structure of tipranavir is different from previous inhibitors, its contact residues for HIV-1 protease share many similarities with other HIV protease inhibitors. Tipranavir inhibits the replication of $\mathrm{HIV}-1$ isolates, with $\mathrm{EC}_{50}$ ranging from $30-70 \mathrm{nM}$. The dosage is tipranavir $500 \mathrm{mg}$ plus ritonavir $200 \mathrm{mg}$ orally, twice a day. However, the adverse effects of tipranavir are more severe than those of other protease inhibitors. Intracranial hemorrhage and decompensated hepatitis have been reported to associate with tipranavir. ${ }^{39}$ The most common clinically significant tipranavir resistance mutations are 32I, 33F, 46IL, 47VA, 54VAM, 82TL, and 84V. 


\section{Darunavir}

Darunavir (brand name: Prezista), approved in 2006, is the latest HIV protease inhibitor on the market. The hydrogen bonds that darunavir forms with the backbone of the HIV protease slow down the development of drug resistance..$^{40}$ The structure of darunavir is very similar to that of amprenavir, the only difference being that in darunavir, the P2 group bis-tetrahydrofuran replaces the tetrahydrofuran group of amprenavir. This change allows darunavir to have more hydrogen bonds with the Asp 29 residues of HIV protease. ${ }^{41}$ The $\mathrm{EC}_{50}$ of darunavir is as low as $1-2 \mathrm{nM}$, and darunavir can inhibit both HIV-1 and HIV-2 with high potency. ${ }^{27,42}$ Furthermore, darunavir or atazanavir coadministrated with ritonavir is a current first-line antiretroviral regimen..$^{43}$ The dosage is darunavir $800 \mathrm{mg}$ plus ritonavir $100 \mathrm{mg}$ orally, once a day. Only minor darunavir-associated toxicities, such as rash, diarrhea, nasopharyngitis, and nausea, have been observed in some patients. ${ }^{44}$ The most common clinically significant darunavir resistance mutations are 32I, 33F, 47VA, $50 \mathrm{~V}, 54 \mathrm{LM}, 76 \mathrm{~V}, 82 \mathrm{~F}$, and $84 \mathrm{~V}$.

\section{The common mechanism of inhibitor binding}

Most current HIV protease inhibitors were designed to mimic the substrate transition state. The hydroxyl group of the inhibitor interacts with the carboxyl group of the protease active site residues, Asp 25 and Asp 25', by hydrogen bonds. The inhibitor-contacting residues of HIV protease are relatively conserved, including Gly 27, Asp 29, Asp 30, and Gly 48, but the accumulation of drug-resistance mutations alters the structure of HIV protease and causes treatment failure.

The major effort in inhibitor development is to modify the available HIV protease inhibitors, which inevitably causes the new inhibitors to inherit structural similarity with previous inhibitors. There have been many attempts to further modify current inhibitors; some have succeeded, while others have not shown significant benefits. A series of compounds has been tested to optimize the P1 group of lopinavir, but none of them has shown significant benefits over the original lopinavir. ${ }^{45}$ The successful examples of modified inhibitors are darunavir and lopinavir, which were modified from amprenavir and ritonavir, respectively.

\section{New designs of HIV protease inhibitors}

It is possible to optimize the chemical structure of HIV protease inhibitors to avoid side effects. For example, not all HIV-1 proteases cause dyslipidemia, such as atazanavir. ${ }^{34}$
Similarly, atazanavir does not inhibit glucose uptake as amprenavir, lopinavir, and ritonavir do. ${ }^{46}$ GS-8374, developed by Gilead Sciences, Inc., (Foster City, CA, USA), is a modification of TMC-126 (darunavir analog). ${ }^{47}$ This new inhibitor does not affect insulin-stimulated glucose uptake or peripheral glucose disposal, which is similar to atazanavir. ${ }^{48}$ A possible explanation may be that the diethylphosphonate moiety modification on the P1 benzyl group of darunavir functions similarly to the large phenylpyridyl P1 group of atazanavir, to repel off-target molecules.

Designing inhibitors based on the substrate envelope of HIV protease is a recent strategy for developing HIV protease inhibitors. The substrate envelope is the overlapping volume occupied by multiple substrates. Based on this theory, a substrate-based HIV protease inhibitor, RO1, has been designed and synthesized. ${ }^{49}$ The results demonstrated that RO1 has five- to tenfold higher inhibitory efficacy than current HIV protease inhibitors. ${ }^{49}$ Among the current FDAapproved HIV protease inhibitors, only tipranavir forms hydrogen bonds with the flap residues of the protease. In contrast, RO1 forms more hydrogen bonds with HIV protease, which enhances the binding energy and is also a step toward a greater diversity of binding patterns. However, the contact residues of RO1 follow the old pattern, and no novel contact has been established. The difficulty of complete binding pattern alteration might be due to the inhibitor design concept of substrate mimics. To develop new scaffolds of HIV protease inhibitors, it is necessary to adopt drug discovery strategies other than substrate mimics.

New scaffolds of HIV protease inhibitors might be a possible solution to eliminate drug side effects. After extensive modifications and tests, the leading new scaffolds might become the next generation of HIV protease inhibitors possessing novel chemical structures and alternative binding patterns to HIV protease. However, it is very risky to explore new structures of inhibitors. One reason is that the new scaffolds are less likely to be superior to the well-established older scaffolds. The structure of mozenavir, which was developed based on the cyclic urea scaffold, is highly dissimilar from other HIV protease inhibitors. ${ }^{50}$ However, the clinical studies showed that mozenavir did not show significant advantages compared with other protease inhibitors on the market, and therefore Gilead Sciences, Inc., stopped the development of mozenavir. ${ }^{51}$ The other reason is that new scaffolds may cause severe adverse effects without thorough clinical studies. The relatively different structure of tipranavir, not only generates altered binding profiles but also, causes the unpredictable side effects, such as hemorrhage. Based on the above summary, 
the nonpeptidomimetic HIV protease inhibitors seem to have a higher tendency to cause severe adverse effects.

Several new HIV protease inhibitors are undergoing clinical trials. GlaxoSmithKline's new inhibitor, CTP-518, was developed by replacing some hydrogen atoms of atazanavir with deuterium, which enables CTP-518 to inherit the once-daily dosage of atazanavir but slows down the drug metabolism. ${ }^{52}$ Another new inhibitor is SPI-256 (Figure 2), developed by Sequoia Pharmaceuticals (Gaithersburg, MD, USA), which is based on the darunavir scaffold. ${ }^{53}$ Rather than coadministration with ritonavir, SPI-256 may be boosted using with Sequoia Pharmaceuticals' own pharmacokinetic enhancer, SPI-452. ${ }^{54}$ This pharmacokinetic enhancer targets cytochrome $\mathrm{P} 4503 \mathrm{~A}$ and boosts the circulating concentration of other protease inhibitors, as ritonavir does, but avoids the side effects caused by ritonavir. ${ }^{55}$

\section{Off-target molecules}

The major metabolic side effects associated with HIV protease inhibitor therapy are lipodystrophy syndrome and insulin resistance. ${ }^{56}$ The possible mechanisms of the metabolic alternations caused by HIV protease inhibitors have been studied. In the following paragraphs, the possible off-target effects of HIV protease inhibitors are discussed. Avoidance of interactions with off-target molecules may be considerations in the future development of new HIV protease inhibitors.

\section{Proteases functioning in SREBP maturation}

It has been demonstrated that most HIV protease inhibitors induce the accumulation of intracellular free cholesterol and lipid. ${ }^{57}$ SREBP-1 is an endoplasmic reticulum (ER)-derived transcription factor that regulates the gene expression involved in lipogenesis. SREBP-1 mediates the effect of insulin on gene expression in adipocytes ${ }^{58}$ and is associated with adipocyte differentiation and metabolism. ${ }^{59}$ In the ER and nuclear envelope, SREBP exists as a full-length membrane-bound precursor. ${ }^{60}$ A two-step sequential proteolytic cleavage activates the membrane-bound SREBP precursors. The first step is a sterol-regulated intramembrane proteolytic step to generate the N-terminal SREBP intermediate, which is processed by a membrane-anchored serine protease. ${ }^{61}$ At the second step, the mature SREBP can be cleaved off by cysteine protease CPP32/SREBP cleavage activity-1 (SCA-1) ${ }^{62}$ or Mch3/SCA-2. ${ }^{63}$ The mature active SREBPs translocate to the nucleus. The inactive SREBP-1 precursor, rather than active SREBP-1, is processed by the ubiquitin-proteasome system. ${ }^{64}$ Studies showed that HIV protease inhibitor therapy caused the sequestration of inactive SREBP-1 at the nuclear membrane level. ${ }^{65,66}$ Nelfinavir and indinavir have demonstrated effects in decreasing active SREBP-1 in the nucleus. ${ }^{65,67}$ The SREBP-1 sequestration prevents activation of the expression of downstream transcription factors for adipocyte differentiation. ${ }^{68}$ Furthermore, SREBP-1 associates with the transcriptional regulation of a fat-derived hormone gene, adiponectin. ${ }^{69}$ The effect of HIV protease inhibitors on SREBPs consequently leads to the deficiency of adiponectin. Therefore, adiponectin replacement therapy or adiponectin receptor agonist could ameliorate dyslipidemia, stabilize metabolic function, and prevent brain injury caused by HIV protease inhibitor-based treatment. ${ }^{70,71}$ Therefore, the off-target inhibition of the proteases required for SREBP maturation could cause inactive SREBP sequestration and affect adipocyte differentiation (Figure 3).

\section{Glucose transporter-4}

The insulin resistance observed in patients with HIV protease inhibitor treatment is associated with the blockage of a glucose transporter (Figure 3). Glucose transporter-4 is an insulin-regulated transporter that can be directly inhibited by many HIV protease inhibitors, especially indinavir. ${ }^{72,73}$ The protease inhibitors specifically block the isoform of glucose transporter-4 and show no significant inhibition of glucose transporter-1 and glucose transporter-2. ${ }^{72,73}$ Therefore, the glucose uptake in adipocytes is blocked.

In vitro testing indicated that peptides with an aromatic core flanked by hydrophobic ends acutely inhibited glucose transporter-4 and weakly inhibited glucose transporter-2 but did not inhibit glucose transporter- 1 and $3 .{ }^{74}$ Those peptides represent the structure characteristic of currently approved HIV protease inhibitors and are also substrates of aspartyl proteases. Therefore, the aromatic core of HIV protease

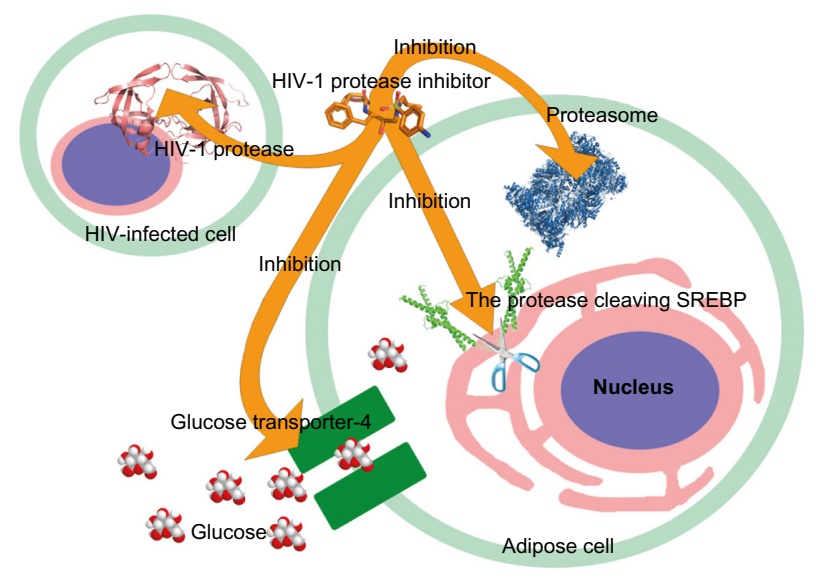

Figure 3 Potential target molecules of the HIV protease inhibitors. 
inhibitors could be the cause of a cross-interaction with glucose transporter-4. Interestingly, atazanavir, carrying an aromatic core, is known for having no effect on glucose uptake. ${ }^{46}$ It is possible that the large phenylpyridyl P1 group of atazanavir, or its azapeptide backbone, disrupts the interaction with glucose transporter-4. Furthermore, atazanavir also differs from other HIV protease inhibitors in the hydrophobicity of the flanked molecule ends. ${ }^{74}$

\section{Proteasome}

The proteasome is, not only a cell apparatus that degrades waste proteins but also, an important regulator in various signal pathways. But proteasome activity can be directly inhibited by some HIV protease inhibitors. ${ }^{75}$ Ritonavir is a very potent proteasome inhibitor at clinically relevant levels. ${ }^{73}$ Ritonavir, as well as nelfinavir and saquinavir, inhibits the proteasomal 20S subunit. ${ }^{76,77}$

Proteasome inhibition could cause metabolic complications (Figure 3). HIV protease inhibitors prevent the proteasomal degradation of apolipoprotein $\mathrm{B}$, and this may cause dyslipidemia, ${ }^{78}$ as apolipoprotein B has a strong effect on the plasma lipid levels. ${ }^{79}$ Saquinavir inhibits proteasome and therefore prevents the activation of NF- $\kappa \mathrm{B} .{ }^{76}$ The inactivation of NF- $\kappa \mathrm{B}$ inevitably leads to cell apoptosis. Furthermore, the inhibition of the proteasome complex that regulates $\mathrm{NF}-\kappa \mathrm{B}$ leads to an adipogenesis reduction. ${ }^{80}$ Furthermore, the proteasome is also responsible for SREBP degradation in the nucleus. ${ }^{64}$ The accumulation of SREBPs in the nucleus constitutively induces the expression of lipogenic and cholesterogenic genes. ${ }^{81}$ HIV protease inhibitor treatment significantly elevates the total cholesterol, low-density lipoprotein cholesterol, and triglycerides in patients. ${ }^{82}$

The toxicity of protease inhibitors also open up a method of drug repositioning. Study has demonstrated that nelfinavir, ritonavir, and saquinavir inhibited protein kinase B (Akt/PKB) and induced caspase-dependent apoptosis. ${ }^{83}$ Darunavir had no significant effect on Akt/PKB, while atazanavir and lopinavir exerted a mild effect. ${ }^{84}$ Among the HIV protease inhibitors tested, nelfinavir showed the great inhibition of Akt activity. ${ }^{83}$ Currently, nelfinavir is undergoing a clinical trial in solid tumors. ${ }^{85}$ Another study showed that the proteasome inhibitors downregulated the activation of Akt. ${ }^{86}$ Thus, inhibition of Akt activity by nelfinavir could be a downstream event of proteasome inhibition. Due to proteasome inhibition, the accumulated unfolded proteins eventually result in ER stress and trigger autophagy. ${ }^{87}$ The ER stress caused by protease inhibitors is one of the rationales for using HIV protease inhibitors as anticancer agents.

\section{Prodrugs in improving bioavailability and reducing side effects}

On one hand, side effects are the consequence of off-target binding. On the other hand, the physiological concentration of drugs is another detrimental factor causing side effects. To optimize the pharmacokinetics and decrease side effects, the development of protease inhibitor prodrugs could improve water solubility and bioavailability. One example is fosamprenavir, the prodrug of amprenavir. Study also showed that conjugating a valine residue to the protease inhibitor, through a hydrolyzable ester bond, improved cell absorption and decreased efflux. ${ }^{88}$ In short, the design of HIV protease inhibitors that maintain potency and eliminate side effects is a long-term exploring process. Before the off-target effects could be eliminated by novel HIV protease inhibitor scaffolds, the development of HIV protease inhibitor prodrugs could decrease dosages and meliorate the side effects.

\section{Future direction in preventing drug side effects}

The identification of off-target mechanisms did not receive sufficient attentions in the past. Determining the potential toxic or benign chemical groups of HIV protease inhibitors helps in the design of newer inhibitors. For example, the P1 group and the two terminal groups of atazanavir may be benign chemical groups and can be considered for incorporation into new HIV protease inhibitors. With the accumulation of knowledge on the toxic chemical groups of HIV protease inhibitors, a "benign chemical group library" for HIV protease inhibitors can be compiled and benefit future drug design studies.

\section{Disclosure}

YW received research supports from the Hunan Provincial Science and Technology Project of China (grant number 2014SK3009) and the Scientific Research Foundation of Hunan Provincial Department of Health (grant number B2013-002). The authors report no other conflicts of interest in this work.

\section{References}

1. UNAIDS. Global Report: UNAIDS Report on the GlobalAIDS Epidemic 2010. Geneva: UNAIDS; 2010.

2. Riddler SA, Haubrich R, DiRienzo AG, et al; AIDS Clinical Trials Group Study A5142 Team. Class-sparing regimens for initial treatment of HIV-1 infection. N Engl J Med. 2008;358(20):2095-2106.

3. Bozzette SA, Ake CF, Tam HK, Chang SW, Louis TA. Cardiovascular and cerebrovascular events in patients treated for human immunodeficiency virus infection. $N$ Engl J Med. 2003;348(8):702-710. 
4. Hruz PW. HIV protease inhibitors and insulin resistance: lessons from in-vitro, rodent and healthy human volunteer models. Curr Opin HIV AIDS. 2008;3(6):660-665.

5. Kotler DP. HIV and antiretroviral therapy: lipid abnormalities and associated cardiovascular risk in HIV-infected patients. J Acquir Immune Defic Syndr. 2008;49 Suppl 2:S79-S85.

6. Soontornniyomkij V, Umlauf A, Chung SA, et al. HIV protease inhibitor exposure predicts cerebral small vessel disease. AIDS. 2014;28(9): 1297-1306.

7. Cameron DW, da Silva BA, Arribas JR, et al. A 96-week comparison of lopinavir-ritonavir combination therapy followed by lopinavirritonavir monotherapy versus efavirenz combination therapy. $J$ Infect Dis. 2008;198(2):234-240.

8. Kolta S, Flandre P, Van PN, et al. Fat tissue distribution changes in HIV-infected patients treated with lopinavir/ritonavir. Results of the MONARK trial. Curr HIV Res. 2011;9(1):31-39.

9. Paton NI, Kityo C, Hoppe A, et al; EARNEST Trial Team. Assessment of second-line antiretroviral regimens for HIV therapy in Africa. $N E n g l$ J Med. 2014;371(3):234-247.

10. Krohn A, Redshaw S, Ritchie JC, Graves BJ, Hatada MH. Novel binding mode of highly potent HIV-proteinase inhibitors incorporating the (R)-hydroxyethylamine isostere. J Med Chem. 1991;34(11): 3340-3342.

11. US Food and Drug Administration. Roche, Fortovase $e^{\circledR}$ (saquinavir). Silver Spring, MD: US Food and Drug Administration; 2003. Available from: http://www.accessdata.fda.gov/drugsatfda_docs/label/2003/20828s015ppi. pdf. Accessed March 25, 2015.

12. Weller IV, Williams IG. ABC of AIDS. Antiretroviral drugs. BMJ. 2001;322(7299):1410-1412.

13. Cameron DW, Japour AJ, Xu Y, et al. Ritonavir and saquinavir combination therapy for the treatment of HIV infection. AIDS. 1999;13(2): 213-224.

14. González de Requena D, Gallego O, de Mendoza C, Corral A, Jiménez-Nácher I, Soriano V. Indinavir plasma concentrations and resistance mutations in patients experiencing early virological failure. AIDS Res Hum Retroviruses. 2003;19(6):457-459.

15. Hresko RC, Hruz PW. HIV protease inhibitors act as competitive inhibitors of the cytoplasmic glucose binding site of GLUTs with differing affinities for GLUT1 and GLUT4. PLoS One. 2011;6(9):e25237.

16. Viraben R, Aquilina C. Indinavir-associated lipodystrophy. AIDS. 1998; 12(6):F37-F39.

17. Kempf DJ, Marsh KC, Kumar G, et al. Pharmacokinetic enhancement of inhibitors of the human immunodeficiency virus protease by coadministration with ritonavir. Antimicrob Agents Chemother. 1997;41(3): 654-660.

18. Zeldin RK, Petruschke RA. Pharmacological and therapeutic properties of ritonavir-boosted protease inhibitor therapy in HIV-infected patients. J Antimicrob Chemother. 2004;53(1):4-9.

19. López Aspiroz E, Cabrera Figueroa SE, Iglesias Gómez A, Valverde Merino MP, Domínguez-Gil Hurlé A. CYP3A4 polymorphism and lopinavir toxicity in an HIV-infected pregnant woman. Clin Drug Investig. 2015;35(1):61-66.

20. Purnell JQ, Zambon A, Knopp RH, et al. Effect of ritonavir on lipids and post-heparin lipase activities in normal subjects. AIDS. 2000;14(1): 51-57.

21. Max B, Sherer R. Management of the adverse effects of antiretroviral therapy and medication adherence. Clin Infect Dis. 2000;30 Suppl 2: S96-S116.

22. Sham HL, Kempf DJ, Molla A, et al. ABT-378, a highly potent inhibitor of the human immunodeficiency virus protease. Antimicrob Agents Chemother. 1998;42(12):3218-3224.

23. Noor MA, Parker RA, O'Mara E, et al. The effects of HIV protease inhibitors atazanavir and lopinavir/ritonavir on insulin sensitivity in HIV-seronegative healthy adults. AIDS. 2004;18(16):2137-2144.

24. Manfredi R, Sabbatani S. Serious, multi-organ hypersensitivity to lopinavir alone, involving cutaneous-mucous rash, and myeloid, liver, and kidney function. AIDS. 2006;20(18):2399-2400.
25. Cresswell FV, Tomlins J, Churchill DR, Walker-Bone K, Richardson D. Achilles tendinopathy following Kaletra (lopinavir/ritonavir) use. Int J STD AIDS. 2014;25(11):833-835.

26. St Clair MH, Millard J, Rooney J, et al. In vitro antiviral activity of 141W94 (VX-478) in combination with other antiretroviral agents. Antiviral Res. 1996;29(1):53-56.

27. Tie Y, Wang YF, Boross PI, et al. Critical differences in HIV-1 and HIV-2 protease specificity for clinical inhibitors. Protein Sci. 2012; 21(3):339-350.

28. Eron J Jr, Yeni P, Gathe J Jr, et al; KLEAN study team. The KLEAN study of fosamprenavir-ritonavir versus lopinavir-ritonavir, each in combination with abacavir-lamivudine, for initial treatment of HIV infection over 48 weeks: a randomised non-inferiority trial. Lancet. 2006;368(9534):476-482.

29. Gathe JC, Wood R, Sanne I, et al. Long-term (120-Week) antiviral efficacy and tolerability of fosamprenavir/ritonavir once daily in therapy-naive patients with HIV-1 infection: an uncontrolled, open-label, single-arm follow-on study. Clin Ther. 2006;28(5):745-754.

30. Judd A, Duong T, Galli L, et al; European Pregnancy and Paediatric HIV Cohort Collaboration (EPPICC) study group in EuroCoord. Post-licensing safety of fosamprenavir in HIV-infected children in Europe. Pharmacoepidemiol Drug Saf. 2014;23(3):321-325.

31. Becker SA. Atazanavir: improving the HIV protease inhibitor class. Expert Rev Anti Infect Ther. 2003;1(3):403-413.

32. Squires K, Lazzarin A, Gatell JM, et al. Comparison of once-daily atazanavir with efavirenz, each in combination with fixed-dose zidovudine and lamivudine, as initial therapy for patients infected with HIV.J Acquir Immune Defic Syndr. 2004;36(5):1011-1019.

33. Murphy RL, Sanne I, Cahn P, et al. Dose-ranging, randomized, clinical trial of atazanavir with lamivudine and stavudine in antiretroviral-naive subjects: 48-week results. AIDS. 2003;17(18):2603-2614.

34. Cahn PE, Gatell JM, Squires K, et al. Atazanavir - a once-daily HIV protease inhibitor that does not cause dyslipidemia in newly treated patients: results from two randomized clinical trials. J Int Assoc Physicians AIDS Care (Chic). 2004;3(3):92-98.

35. Dauchy FA, Lawson-Ayayi S, de La Faille R, et al. Increased risk of abnormal proximal renal tubular function with HIV infection and antiretroviral therapy. Kidney Int. 2011;80(3):302-309.

36. Calza L, Trapani F, Salvadori C, et al. Incidence of renal toxicity in HIV-infected, antiretroviral-naïve patients starting tenofovir/ emtricitabine associated with efavirenz, atazanavir/ritonavir, or lopinavir/ritonavir. Scand J Infect Dis. 2013;45(2):147-154.

37. Rusconi S, La Seta Catamancio S, Citterio P, et al. Susceptibility to PNU-140690 (Tipranavir) of human immunodeficiency virus type 1 isolates derived from patients with multidrug resistance to other protease inhibitors. Antimicrob Agents Chemother. 2000;44(5):1328-1332.

38. Larder BA, Hertogs K, Bloor S, et al. Tipranavir inhibits broadly protease inhibitor-resistant HIV-1 clinical samples. AIDS. 2000;14(13): 1943-1948.

39. Orman JS, Perry CM. Tipranavir: a review of its use in the management of HIV infection. Drugs. 2008;68(10):1435-1463.

40. Lefebvre E, Schiffer CA. Resilience to resistance of HIV-1 protease inhibitors: profile of darunavir. AIDS Rev. 2008;10(3):131-142.

41. TieY, Boross PI, Wang YF, et al. High resolution crystal structures of HIV-1 protease with a potent non-peptide inhibitor (UIC-94017) active against multi-drug-resistant clinical strains. J Mol Biol. 2004;338(2): 341-352.

42. Brower ET, Bacha UM, Kawasaki Y, Freire E. Inhibition of HIV-2 protease by HIV-1 protease inhibitors in clinical use. Chem Biol Drug Des. 2008;71(4):298-305.

43. Panel on Antiretroviral Guidelines for Adults and Adolescents. Guidelines for the Use of Antiretroviral Agents in HIV-1-Infected Adults and Adolescents. Washington, DC: Department of Health and Human Services; 2011.

44. Molina JM, Cohen C, Katlama C, et al; TMC114-C208 Study Group; TMC114-C215 Study Group. Safety and efficacy of darunavir (TMC114) with low-dose ritonavir in treatment-experienced patients: 24-week results of POWER 3. J Acquir Immune Defic Syndr. 2007;46(1):24-31. 
45. Sham HL, Zhao C, Li L, et al. Novel lopinavir analogues incorporating non-Aromatic P-1 side chains - synthesis and structure - activity relationships. Bioorg Med Chem Lett. 2002;12(21):3101-3103.

46. Yan Q, Hruz PW. Direct comparison of the acute in vivo effects of HIV protease inhibitors on peripheral glucose disposal. J Acquir Immune Defic Syndr. 2005;40(4):398-403.

47. He GX, Yang ZY, Williams M, et al. Discovery of GS-8374, a potent human immunodeficiency virus type 1 protease inhibitor with a superior resistance profile. Med Chem Commun. 2011;2(11):1093-1098.

48. Hruz PW, Yan Q, Tsai L, et al. GS-8374, a novel HIV protease inhibitor, does not alter glucose homeostasis in cultured adipocytes or in a healthy-rodent model system. Antimicrob Agents Chemother. 2011; 55(4):1377-1382.

49. Prabu-Jeyabalan M, King NM, Nalivaika EA, Heilek-Snyder G, Cammack N, Schiffer CA. Substrate envelope and drug resistance: crystal structure of RO1 in complex with wild-type human immunodeficiency virus type 1 protease. Antimicrob Agents Chemother. 2006;50(4): $1518-1521$.

50. Ala PJ, DeLoskey RJ, Huston EE, et al. Molecular recognition of cyclic urea HIV-1 protease inhibitors. J Biol Chem. 1998;273(20): 12325-12331.

51. Hoffmann C. ART 2010/2011: The horizon and beyond. In: Hoffmann C, Rockstroh JK, editors. HIV 2010 - A Textbook. Hamburg: Medizin Fokus Verlag; 2010:127-159.

52. Concert Pharmaceuticals. Pharmacokinetics, safety and tolerability of isotopologs of atazanavir (ATV), with pharmacokinetic comparison to reyataz. Available from: http://clinicaltrials.gov/ct2/ show/NCT01458769?term=CTP-518\&rank=1. NLM identifier: NCT01458769. Accessed March 5, 2015.

53. Holland A, Wynne B, Ruff D, Guttendorf R. A first in human study evaluating the safety, tolerability, and pharmacokinetics (PK) of SPI-256, a novel HIV protease inhibitor (PI), administered alone and in combination with ritonavir (RTV) in healthy adult subjects. Poster presented at the 48th Annual ICAAC/46th Annual IDSA Meeting; October 25-28; 2008; Washington, DC.

54. Gulnik SV, Eissenstat M. Approaches to the design of HIV protease inhibitors with improved resistance profiles. Curr Opin HIV AIDS. 2008;3(6):633-641.

55. Gulnik S, Eissenstat M, Afonina E, et al. Preclinical and early clinical evaluation of SPI-452, a new pharmacokinetic enhancer (PKE). Presented at the 16th CROI Conference on Retroviruses and Opportunistic Infections; February 8-12; 2009; Montreal, Qc.

56. Nolan D. Metabolic complications associated with HIV protease inhibitor therapy. Drugs. 2003;63(23):2555-2574.

57. Zhou H, Gurley EC, Jarujaron S, et al. HIV protease inhibitors activate the unfolded protein response and disrupt lipid metabolism in primary hepatocytes. Am J Physiol Gastrointest Liver Physiol. 2006;291(6): G1071-G1080.

58. Kim JB, Sarraf P, Wright M, et al. Nutritional and insulin regulation of fatty acid synthetase and leptin gene expression through ADD1/ SREBP1. J Clin Invest. 1998;101(1):1-9.

59. Fajas L, Schoonjans K, Gelman L, et al. Regulation of peroxisome proliferator-activated receptor gamma expression by adipocyte differentiation and determination factor $1 /$ sterol regulatory element binding protein 1: implications for adipocyte differentiation and metabolism. Mol Cell Biol. 1999;19(8):5495-5503.

60. Sato R, Yang J, Wang X, et al. Assignment of the membrane attachment, DNA binding, and transcriptional activation domains of sterol regulatory element-binding protein-1 (SREBP-1). J Biol Chem. 1994;269(25): 17267-17273.

61. Sakai J, Rawson RB, Espenshade PJ, et al. Molecular identification of the sterol-regulated luminal protease that cleaves SREBPs and controls lipid composition of animal cells. Mol Cell. 1998;2(4):505-514.

62. Wang X, Pai JT, Wiedenfeld EA, et al. Purification of an interleukin-1 beta converting enzyme-related cysteine protease that cleaves sterol regulatory element-binding proteins between the leucine zipper and transmembrane domains. J Biol Chem. 1995;270(30):18044-18050.
63. Pai JT, Brown MS, Goldstein JL. Purification and cDNA cloning of a second apoptosis-related cysteine protease that cleaves and activates sterol regulatory element binding proteins. Proc Natl Acad Sci US A. 1996;93(11):5437-5442.

64. Hirano Y, Yoshida M, Shimizu M, Sato R. Direct demonstration of rapid degradation of nuclear sterol regulatory element-binding proteins by the ubiquitin-proteasome pathway. J Biol Chem. 2001;276(39): 36431-36437.

65. Caron M, Auclair M, Vigouroux C, Glorian M, Forest C, Capeau J. The HIV protease inhibitor indinavir impairs sterol regulatory elementbinding protein-1 intranuclear localization, inhibits preadipocyte differentiation, and induces insulin resistance. Diabetes. 2001;50(6): 1378-1388.

66. Guan M, Fousek K, Chow WA. Nelfinavir inhibits regulated intramembrane proteolysis of sterol regulatory element binding protein-1 and activating transcription factor 6 in castration-resistant prostate cancer. FEBS J. 2012;279(13):2399-2411.

67. Tran H, Robinson S, Mikhailenko I, Strickland DK. Modulation of the LDL receptor and LRP levels by HIV protease inhibitors. J Lipid Res. 2003;44(10):1859-1869.

68. Bastard JP, Caron M, Vidal H, et al. Association between altered expression of adipogenic factor SREBP1 in lipoatrophic adipose tissue from HIV-1-infected patients and abnormal adipocyte differentiation and insulin resistance. Lancet. 2002;359(9311):1026-1031.

69. Rahmouni K, Sigmund CD. Id3, E47, and SREBP-1c: fat factors controlling adiponectin expression. Circ Res. 2008;103(6):565-567.

70. Xu A, Yin S, Wong L, Chan KW, Lam KS. Adiponectin ameliorates dyslipidemia induced by the human immunodeficiency virus protease inhibitor ritonavir in mice. Endocrinology. 2004;145(2):487-494.

71. Pepping JK, Otvos L, Surmacz E, Gupta S, Keller JN, Bruce-Keller AJ. Designer adiponectin receptor agonist stabilizes metabolic function and prevents brain injury caused by HIV protease inhibitors. J Neuroimmune Pharmacol. 2014;9(3):388-398.

72. Murata H, Hruz PW, Mueckler M. The mechanism of insulin resistance caused by HIV protease inhibitor therapy. J Biol Chem. 2000;275(27): 20251-20254.

73. Murata H, Hruz PW, Mueckler M. Indinavir inhibits the glucose transporter isoform Glut4 at physiologic concentrations. AIDS. 2002;16(6): 859-863.

74. Hertel J, Struthers H, Horj CB, Hruz PW. A structural basis for the acute effects of HIV protease inhibitors on GLUT4 intrinsic activity. J Biol Chem. 2004;279(53):55147-55152.

75. André P, Groettrup M, Klenerman P, et al. An inhibitor of HIV-1 protease modulates proteasome activity, antigen presentation, and $\mathrm{T}$ cell responses. Proc Natl Acad Sci U S A. 1998;95(22):13120-13124.

76. Pajonk F, Himmelsbach J, Riess K, Sommer A, McBride WH. The human immunodeficiency virus (HIV)-1 protease inhibitor saquinavir inhibits proteasome function and causes apoptosis and radiosensitization in non-HIV-associated human cancer cells. Cancer Res. 2002;62(18): 5230-5235.

77. Hamel FG, Fawcett J, Tsui BT, Bennett RG, Duckworth WC. Effect of nelfinavir on insulin metabolism, proteasome activity and protein degradation in HepG2 cells. Diabetes Obes Metab. 2006;8(6):661-668.

78. Liang JS, Distler O, Cooper DA, et al. HIV protease inhibitors protect apolipoprotein B from degradation by the proteasome: a potential mechanism for protease inhibitor-induced hyperlipidemia. Nat Med. 2001;7(12):1327-1331.

79. Steinberg D, Carew TE, Fielding C, et al. Lipoproteins and the pathogenesis of atherosclerosis. Circulation. 1989;80(3):719-723.

80. De Barros S, Zakaroff-Girard A, Lafontan M, Galitzky J, Bourlier V. Inhibition of human preadipocyte proteasomal activity by HIV protease inhibitors or specific inhibitor lactacystin leads to a defect in adipogenesis, which involves matrix metalloproteinase-9. J Pharmacol Exp Ther. 2007;320(1):291-299.

81. Parker RA, Flint OP, Mulvey R, et al. Endoplasmic reticulum stress links dyslipidemia to inhibition of proteasome activity and glucose transport by HIV protease inhibitors. Mol Pharmacol. 2005;67(6):1909-1919. 
82. Mulligan K, Grunfeld C, Tai VW, et al. Hyperlipidemia and insulin resistance are induced by protease inhibitors independent of changes in body composition in patients with HIV infection. J Acquir Immune Defic Syndr. 2000;23(1):35-43.

83. Gills JJ, Lopiccolo J, Tsurutani J, et al. Nelfinavir, A lead HIV protease inhibitor, is a broad-spectrum, anticancer agent that induces endoplasmic reticulum stress, autophagy, and apoptosis in vitro and in vivo. Clin Cancer Res. 2007;13(17):5183-5194.

84. Capel E, Auclair M, Caron-Debarle M, Capeau J. Effects of ritonavirboosted darunavir, atazanavir and lopinavir on adipose functions and insulin sensitivity in murine and human adipocytes. Antivir Ther. 2012; 17(3):549-556.
85. National Cancer Institute (NCI). Nelfinavir in treating patients with metastatic, refractory, or recurrent solid tumors. Available from: https://clinicaltrials.gov/ct2/show/NCT00436735. NLM identifier: NCT00436735. Accessed March 25, 2015.

86. Inoue T, Shiraki K, Fuke H, et al. Proteasome inhibition sensitizes hepatocellular carcinoma cells to TRAIL by suppressing caspase inhibitors and AKT pathway. Anticancer Drugs. 2006;17(3):261-268.

87. Yorimitsu T, Nair U, Yang Z, Klionsky DJ. Endoplasmic reticulum stress triggers autophagy. J Biol Chem. 2006;281(40):30299-30304.

88. Roche D, Greiner J, Aubertin AM, Vierling P. Synthesis and in vitro biological evaluation of valine-containing prodrugs derived from clinically used HIV-protease inhibitors. Eur J Med Chem. 2008;43(7): 1506-1518.

\section{Publish your work in this journal}

HIV/AIDS - Research and Palliative Care is an international, peerreviewed open-access journal focusing on advances in research in HIV, its clinical progression and management options including antiviral treatment, palliative care and public healthcare policies to control viral spread. The journal welcomes original research, basic science, clinical \& epidemiological studies, reviews \& evaluations, expert opinion \& commentary, case reports \& extended reports. The manuscript management system is completely online and includes a very quick and fair peer-review system. Visit http://www.dovepress.com/ testimonials.php to read real quotes from published authors.

Submit your manuscript here: http://www.dovepress.com/hivaids---research-and-palliative-care-journal 